\title{
SOBRE LAS CONSTRUCCIONES INDEFINIDAS PARA HUMANOS EN ESPAÑOL*
}

Eduardo Tadeu Roque Amaral * *

Resumen: El objetivo de este trabajo es identificar y analizar elementos como persona, individuo, fulano, tal o cual, etc. en contextos típicos de pronombres indefinidos. La base teórica está constituida por estudios sobre esta clase de palabras y sobre los nombres generales. Los datos analizados provienen del CORPES XXI y de test de aceptabilidad. Los resultados permiten observar grupos de elementos formados por nombres generales, por otros nombres más periféricos y por construcciones no nominales, además de verificar tendencias según la clasificación temática de los textos del corpus.

Palabras clave: Pronombres indefinidos. Nombres generales. Lengua española.

\section{INTRODUCCIÓN}

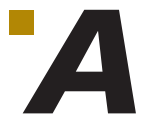

1 tratar de los cuantificadores indefinidos, la Nueva Gramática de la Lengua Española (NGLE) distingue diferentes grupos. Unidades como alguien, alguno, nadie y ninguno se incluyen en el grupo de los cuantificadores existenciales, porque aluden a la existencia de una entidad, cuya caracterización puede o no ser restringida mediante complementos. Estos elementos se distribuyen entre positivos y negativos, clasificación también presentada por Martínez (1989, p. 84). La forma cualquiera pertenece al grupo de indefinidos de indistinción o de elección libre, porque se refiere a una entidad

\footnotetext{
Agradezco a Wiltrud Mihatsch (Universidad de Tubinga) y a Karolin Moser (Universidad Nacional de Córdoba) los comentarios y sugerencias. Agradezco también a la Asociación de Universidades Grupo Montevideo (AUGM), en el marco del Programa Escala Docente, la posibilidad de recolección de los datos en la Universidad Nacional de Córdoba. Al profesor Juan José Rodríguez le agradezco la acogida en la ciudad y la colaboración en la aplicación de los cuestionarios. A Vitor Lamartine le agradezco el auxilio en la organización de los datos.

** Universidade Federal de Minas Gerais (UFMG) - Belo Horizonte - MG - Brasil. E-mail: eduamaralbh@uol.com.br
} 
elegida arbitrariamente entre otras ${ }^{1}$ (NGLE, § 20.3a, p. 1457). Sánchez López (1999) incluye las unidades anteriores entre los cuantificadores propios indefinidos, siendo alguien y nadie no universales y cualquiera universal.

Al observar el funcionamiento de los elementos citados, se verifica que el hablante emplea el indefinido alguien cuando desea hablar de la existencia de un individuo cuyo nombre no se sabe, no se quiere o no se puede nombrar. De acuerdo con la NGLE, sería un contexto presentativo, en el que se menciona la existencia de una persona cuya naturaleza se desconoce (NGLE, § 20.3e, p. 1458). El pronombre nadie se utiliza en sentido opuesto, para hablar de la inexistencia de una persona. Con cualquiera, se puede tener una interpretación existencial (Pregúntale a cualquiera) o universal (Eso le puede suceder a cualquiera). Aún según la NGLE, cualquiera se interpreta como cualquier persona cuando no se puede recuperar un nombre común que restrinja el dominio de la cuantificación (NGLE, § 20.4ñ, p. 1471).

En todos estos casos, se hace abstracción del sexo de la persona y de cualquier otra característica suya, como profesión, grado de parentesco, etc., lo que acerca estos pronombres a los llamados nombres generales, unidades lingüisticas con las que también se abstraen la casi totalidad de características del referente (MIHATSCH, 2015). En español, forman parte de este grupo palabras como persona, individuo, ser humano, sujeto, etc., que suelen recibir definiciones bien semejantes en los diccionarios. Compárense, a propósito, las siguientes definiciones del Diccionario de la Real Academia Española (DRAE, 2016):

1. individuo 3. m. y f. coloq. Persona cuyo nombre y condición se ignoran o no se quieren decir.

2. persona 2. f. Hombre o mujer cuyo nombre se ignora o se omite.

3. sujeto 3. m. Persona cuyo nombre se ignora o no se quiere decir.

Con base en lo expuesto, este trabajo tiene como objetivo identificar y analizar un conjunto de nombres del español, con enfoque en la variedad argentina, que permite al hablante hacer referencia a la existencia (o inexistencia) de un ser humano sin la identificación de sexo o de otra característica. Dicho de otro modo, son elementos que tienden a presentar únicamente el rasgo [+humano] en los contextos aquí seleccionados. Además de observar los aspectos semántico-léxicos, se busca verificar las posibles tendencias de empleo de cada nombre según el tema del texto en que cada construcción se encuentra.

En términos sintácticos, los indefinidos alguien y nadie ocupan la posición de un sintagma nominal, así como las construcciones seleccionadas para este trabajo. Esto no significa que estos indefinidos no puedan usarse antecedidos por un, como muestra Larrivée (2009), para quien la construcción atípica de un pronombre indefinido acompañado por determinante posee una semántica regular y una sintaxis paradójica (LARRIVÉE, 2009, p. 14), ya que esta categoría de pronombre supondría ser la proyección máxima del sintagma ${ }^{2}$. Los indefinidos también

1 La NGLE aun reconoce los indefinidos evaluativos (poco, un poco, un tanto, etc.), que no se relacionan con los elementos seleccionados para este trabajo (NGLE, § 20.3a, p. 1457). Además, los Ilamados cuantificadores existenciales pueden ser, según la NGLE, pronombres, adjetivos o adverbios, pero a este texto conciernen únicamente las formas que tienen carácter pronominal.

2 Los ejemplos 1., 2. y 3., retirados del CORPES XXI, presentan indefinidos antecedidos por un, todos de obras de ficción: 
pueden estar modificados por diferentes tipos de sintagmas (aunque con determinadas restricciones), lo que también es posible con los nombres de este estudio. Sin embargo, como se explicará más adelante en la metodología, se analizan solamente los casos sin modificadores.

\section{Fundamentación TEÓRICA}

Este trabajo se enmarca en un conjunto de estudios sobre los llamados nombres generales, buscando relacionar los usos de esta categoría de palabras en construcciones indefinidas con otros elementos de la lengua que pueden desempeñar funciones equivalentes. En los estudios lingüísticos de las últimas décadas, hay varios trabajos que hacen algún tipo de referencia a esta categoría de palabras, la cual sólo hace pocos años empezó a recibir una atención especial por parte de los lingüistas. Entre los trabajos que mencionan este grupo de nombres, se pueden citar los que se dedican a la cohesión léxica (HALLIDAY; HASAN, 1995 [1976]; FRANCIS, 1994; SCHMID, 2000); los que consideran los presupuestos de la lingüística de corpus (MAHLBERG, 2005); los que se ocupan del contraste lingüístico (KOCH; OESTERREICHER, 2007); y los que investigan los procesos de gramaticalización y de formación de pronombres (HASPELMATH, 1997; HEINE; KUTEVA, 2004; HEINE; SONG, 2010, 2011; GIACALONE RAMAT; SANSÒ, 2007, 2011).

Dos elementos representativos de esta categoria y a la vez prototípicos de los que llevan el rasgo [+humano] y [-humano] son, respectivamente, persona y cosa. Respecto a estas unidades lingüísticas, gramáticos antiguos llegaron a observar su afinidad con los indefinidos. De la Torre y Ocón (1728), por ejemplo, los incluye en el conjunto de los que llamó pronombres impropios o indefinidos.

Gonzalo Correas (1984 [1627], p. 140) también llegó a hablar del carácter pronominal de cosa: "deste nonbre cosa podemos dezir ansi mesmo, que sirve de neutro, i de pronombre porque neutralmente se habla por cosa, aunque es femenina su terminazion, i sirve de pronombre a todo". Y sobre las formas para seres animados, se lee:

Lo mesmo es en kastellano criatura, cria, $i$ persona, que con forma feminina, sin distinghir neutralmente dizen macho i hembra: Fulana parió una criatura muerta: Tiene al pecho una criatura; las ovexas con sus crias: las personas de este lugar (p. 140).

El mismo autor atribuye a la gente "del vulgo" construcciones como un cómo se llama, el cómo se llama, un cómo se llamó, que serían utilizadas "quando [las personas del vulgo] no se acuerdan, como se llama la cosa, o persona, que quieren nonbrar" (CORREAS, 1984, p. 141) . $^{3}$

1. la soledad me empieza a carcomer tanto como la ausencia de un alguien (VISNEVETSKY, B. Auto de fe. In: BAILE CORTÉS, I. [et al.]: Teatro. Piezas breves. Alumnos RESAD. Curso 2004/2005. Madrid: Fundamentos, 2005.)

2. Esas paredes heladas, decoradas por un nadie... (MUÑOZ, A. Las mujeres entre los hielos. In: MUÑOZ, A.; CANO, L. LÓPEZ MEDIN, S.: Teatro 7. Buenos Aires: Instituto Nacional del Teatro, 2007.)

3. Se lo veía señorial y prepotente, más apropiado para un embajador que para un cualquiera (ARGEMÍ, R. Siempre la misma música. Sevilla: Algaida, 2006.)

3 El gramático también identifica formas marcadas diatópica o diastráticamente:

En Aragón dizen el aquel, un aquel por lo mesmo. Los rrusticos dizen el quillotro, o quillotre, un quillotro, i forman verbos 
Aparte de las formas que frecuentemente se incluyen en el conjunto de los indefinidos, como nada, algo, nadie, alguien, etc., Alvar y Pottier (1983, p. 150) agregan: "Se emplean, o se emplearon, como indefinidos una serie de voces o de sintagmas habilitados como tales: citano, cualque cosa, dello, fulano, gente, gentes humanas, no sé qué, persona, tal, todo el mundo, todo viviente, uno, etc."

Se ve claramente que no es reciente la relación establecida entre los indefinidos y cierto grupo de sustantivos, pero faltan estudios sistemáticos sobre el conjunto de unidades que pueden formar parte de este grupo, sobre todo teniendo en cuenta el corpus de la lengua contemporánea. Por lo tanto, contribuir para ello es uno de los propósitos de este trabajo.

\section{Los nombres generales}

Entre las publicaciones recientes que se ocupan específicamente de la categoría de los nombres generales están Amaral y Ramos (2014), que analiza datos del portugués brasileño, y Mihatsch (2015), que investiga el comportamiento de estos nombres en datos del alemán y del francés. Unidades lingüísticas como individuo, persona y ser humano o sus correspondientes en otras lenguas han sido incluidas en esta categoría de palabras. En ese caso, todas presentan el rasgo [+humano] y en general son elementos que nacen de contextos eruditos, especialmente jurídicos, teológicos, filosóficos y didácticos, en los que se hace abstracción del sexo (MIHATSCH, 2015, p. 62).

Aún de acuerdo con Mihatsch (2015), cuando el hablante se refiere a un individuo específico, emplea frecuentemente el nombre propio. Si éste no está disponible, utiliza casi siempre un nombre que identifica el sexo del referente (hombre, mujer, hijo etc.) o un sintagma con nombres relacionales (mi colega, tu amigo, su madre), que señalan relaciones familiares o sociales. De acuerdo con la autora, la especificación del sexo es el rasgo más relevante en la categorización del individuo y en la formación de estereotipos, lo que explicaría la diferenciación de sexo en la mayoría de los nombres de pila y de las fórmulas de tratamiento (MIHATSCH, 2015, p. 61).

Sin embargo, para la referencia a un conjunto de individuos sin identificación de sexo o a una persona inespecífica, los nombres generales son de fundamental importancia. Aparte de unidades estudiadas como individuo, persona o ser humano, se observa una serie de nombres para humanos, que, de acuerdo con la autora, son morfológicamente complejos y poco frecuentes, originados de contextos teológicos o filosóficos. Se trata de elementos que serian arcaicos, empleados de forma lúdica o marcados estilísticamente, como bipede, mortal, alma, etc. Si bien pueden ser unidades con baja frecuencia, se cree que son importantes para el conocimiento del conjunto de nombres para humanos, especialmente si se tienen en cuenta los contextos y los registros en que se realizan.

\section{La semántica de los indefinidos}

El conjunto de construcciones que se analizan en este trabajo se acercan semántica y pragmáticamente al empleo de los pronombres indefinidos. De hecho, si se observa

quillotrar, como se llamar, i feminino quillotra, la como se llama (CORREAS, 1984 [1627], p. 141). 
el comportamiento de algunos nombres generales frecuentes en otras lenguas, se ve una gran afinidad con esta clase de palabras. Haspelmath (1997) observa que no es fácil diferenciar nombres generales de pronombres indefinidos basados en tales nombres (p. ej. something vs. some thing). Además, destaca el autor que

[...] indefinite pronouns are sometimes derived from generic ontological-category nouns such as "person", "thing", "place", "time”, "manner". These generic nouns are very similar in meaning to indefinite pronouns like "somebody", "something", "somewhere", "sometime", "somehow", and languages may make use of indefinite noun phrases consisting only of such generic nouns to express what other languages express by means of indefinite pronouns (HASPELMATH, 1997, p. 52-53).

Con base en el estudio de Haspelmath (1997), se puede hablar de tres series de indefinidos en español: alg-; $n$ - y -quier. Serian ejemplos de la primera los pronombres alguien, algo, alguno; de la segunda, quienquiera y cualquiera; y de la tercera nadie, nada y ninguno. Como determinantes, tendriamos: algún(a), ningún(a) y cualquier(a). En este artículo, se estudian los casos representativos de las tres series, tanto en la interpretación genérica como en otras discutidas por el autor en su mapa semántico, como inespecífica, negación y elección libre.

Respecto a la interpretación genérica, al tratar de los diferentes empleos del artículo indefinido un, Leonetti (1999, p. 877) diferencia las interpretaciones genéricas de las inespecíficas y afirma que "ambas lecturas son no referenciales y son originadas por las características del entorno oracional, pero se distinguen porque la interpretación genérica exige que el SN sea tema en una estructura de juicio categórico". Un enunciado como Un granjero cuida el ganado todos los días podría tener, aparte de una lectura específica, una genérica, que se interpretaría como "De un granjero, en general, se puede decir que cuida el ganado todos los dias" y otra inespecífica, que se interpretaría como "Todos los dias hay un granjero que se ocupa del ganado" (p. 877). Por lo tanto, en este trabajo, se observa la propiedad de ser tema y la de incluir un predicado con un juicio categórico sobre el ser humano.

En lo que se refiere a la interpretación inespecífica, hay un gran número de trabajos sobre el tema, los cuales se dedican a las diferentes estrategias que posibilitan tal lectura (LEONETTI, 1999, 2012; NGLE, 2009). Para este estudio, se considera un enunciado inespecífico cuando el locutor no se refiere a un individuo particular, sino a un potencial que puede satisfacer cierta condición, en el caso, la condición de ser humano. En otras palabras, diferentemente de la interpretación específica, el locutor no presupone la identificabilidad del referente.

En los casos de negación, el locutor niega la existencia de un individuo, lo que se puede hacer por medio de una negación directa (No vi a ninguna persona en el despacho) o indirecta (No creo que ninguna persona sea capaz de decirlo) (HASPELMATH, 1997, p. 31). Por fin, considerando la interpretación de elección libre, el locutor explicita que la identificación del individuo es irrelevante o indiferente (LEONETTI, 2012).

Los tipos de interpretación seleccionados se justifican por el hecho de que, como señalado por Haspelmath (1997), los pronombres indefinidos nacen de funciones expresivas enfáticas, especialmente como el pronombre negativo o como el pronombre indefinido de elección libre. Amaral y Mihatsch $(2016$, p. 3) 
destacan, además, que sobre todo en estas funciones, se observa la construcción de creaciones nuevas, que atestiguan la existencia de (casi-)sinónimos, como ocurre en el caso del francés nul, personne, aucun en la función negativa y n’importe qui, quelconque, quiconque, qui que ce soit para la función de elección libre.

\section{Procedimientos metodológicos}

En este trabajo, se analizan fundamentalmente datos del Corpus del Español del Siglo XXI (CORPES XXI), complementados, siempre que posible, con parte de los resultados de un test de aceptabilidad aplicado a hablantes nativos del español. A continuación, se explican con más detalles estas dos fuentes de datos, así como los procedimientos empleados para el análisis.

El CORPES XXI es un corpus en línea formado por textos de libro y de prensa, el cual presenta un 30\% de formas procedentes de España y un 70\% de formas procedentes de América (RAE, 2013). Para este artículo, se restringió la búsqueda a los datos de Argentina y a los siguientes contextos sintácticos: $\left[_{\mathrm{SN}}\right.$ un / algún / ningún / cualquier $\left.[\mathrm{N}] \varnothing\right]$, donde $[\mathrm{N}]$ representa uno de los siguientes nombres, seleccionados a partir de los estudios comentados en la sección anterior: alma, bipede, ciudadano, fulano, humano, individuo, mortal, persona, prójimo, quidam, ser humano, semejante, sujeto, tipo y viviente. Se incluyó también una consulta a formas no nominales, citadas por diferentes gramáticos o lingüistas, como no sé quién, tal o cual, un cómo se llama/llamó, un aquel, un don nadie y no importa quién.

Los contextos citados en el párrafo anterior posibilitan analizar enunciados con lecturas genéricas, inespecíficas, de negación y de elección libre. Se excluyen, por lo tanto, lecturas específicas (Hay una persona de mi familia que quiere hablar contigo) y cardinales (Una persona más completa el equipo) (LEONETTI, 1999, 2012, p. 293). Se eliminó también la posibilidad de adjetivo antepuesto al nombre, que puede llevar a un cambio en la especificidad (LEONETTI, 2012, p. 298) y de cualquier modificador pospuesto.

En la composición de la muestra, se consideró asimismo la clasificación temática con la cual está organizado el CORPES XXI (Cuadro 1). Esta clasificación permite observar tendencias en el empleo de los nombres y contrastar los datos del corpus con algunos resultados de los juicios de aceptabilidad de nativos del español.

Cuadro 1 - Clasificación temática del CORPES XXI

\begin{tabular}{|l|c|}
\hline No ficción & Ficción \\
\hline a.Actualidad, ocio y vida cotidiana & a. Guion \\
\hline b.Artes, cultura y espectáculos & b. Novela \\
\hline c. Ciencias sociales, creencias y pensamiento & c. Relato \\
\hline d. Ciencias y tecnología & d. Teatro \\
\hline e. Política, economía y justicia & \\
\hline f. Salud & \\
\hline
\end{tabular}


Con el fin de observar la aceptabilidad de los nombres generales en diferentes tipos de enunciados, se elaboraron, en el marco de una investigación de mayor envergadura, 6 modelos de cuestionarios, cada uno con 50 enunciados, que fueron aplicados en 2015 en la Facultad de Lenguas de la Universidad Nacional de Córdoba (UNC) ${ }^{4}$. Los informantes eran todos nativos de español, estudiaban en la UNC, tenían entre 18 y 50 años y habían vivido la mayor parte de la vida en la Provincia de Córdoba. Durante la aplicación de los cuestionarios, tenían que evaluar los diferentes enunciados con los nombres generales según una escala Likert de 1 a 5, conforme la siguiente clasificación:

1: uso totalmente aceptable

2: uso aceptable

3: uso más o menos aceptable

4: uso poco aceptable

5: uso no aceptable

En este estudio, se analizan los resultados obtenidos para los enunciados que se relacionan con el objetivo anteriormente expuesto, considerando el promedio de 30 respuestas. En la lectura de los ejemplos de la sección siguiente, los valores entre (1) y (5) representan, respectivamente, una escala de mayor a menor aceptabilidad.

El análisis que sigue se presenta de la siguiente forma: inicialmente, se discuten los casos de los nombres que han sido incluidos en el conjunto de los nombres generales por estudios anteriores: humano; individuo; persona; ser humano; sujeto. En general, son más frecuentes que los demás y reciben definiciones semejantes en el DRAE, como destacado anteriormente. En seguida, se analizan los casos de nombres que también ocurren en contextos indefinidos, pero, como se verá, tienen un comportamiento más marginal en contraste con el grupo anterior: alma, ciudadano, mortal, semejante, tipo y fulano. Por fin, se discuten los casos de construcciones encontradas en el corpus que también se emplean para la referencia a un ser humano cualquiera, pero no llevan un nombre en su constitución.

\section{ANÁlisis DE LOS DATOS}

\section{1er grupo: construcciones con los nombres generales}

Considerando los contextos sintácticos seleccionados para este trabajo, se obtienen, en los datos del CORPES XXI/Argentina, 535 ocurrencias con nombres generales, distribuidas según la Tabla 1: 
Tabla 1 - Distribución de los nombres generales según el determinante que los antecede en SSNN indefinidos

\begin{tabular}{|l|c|c|c|c|c|}
\hline & un(a) & algún(a) & ningún(a) & cualquier & Total \\
\hline persona & $300^{5}$ & 11 & 8 & 50 & 369 \\
\hline ser humano & 54 & 1 & 11 & 19 & 85 \\
\hline individuo & 49 & 1 & 4 & 1 & 55 \\
\hline humano & 11 & 0 & 1 & 0 & 12 \\
\hline sujeto & 14 & 0 & 0 & 0 & 14 \\
\hline Total & $\mathbf{4 2 8}$ & $\mathbf{1 3}$ & $\mathbf{2 4}$ & $\mathbf{7 0}$ & $\mathbf{5 3 5}$ \\
\hline
\end{tabular}

Entre todos los nombres, persona es el más frecuente en la muestra. Se puede decir que es el más prototípico entre los nombres generales, del mismo modo que pessoa lo es para el portugués (AMARAL; RAMOS, 2014). Considerando los empleos indefinidos, se observa una mayor frecuencia en textos no ficcionales, principalmente en los temas Ciencias y Tecnología (1) y Politica, economía y justicia (2), ejemplificados a continuación:

(1) También se sostiene que las propuestas de los abogados del hipertexto no tienen en cuenta qué es lo primero que debe hacer una persona cuando se sienta frente a un programa (PISCITELLI, A. Ciberculturas 2.0. En la era de las máquinas inteligentes. Buenos Aires: Paidós, 2002) ${ }^{6}$.

(2) Requisar a una persona en la calle, hacerle algunas preguntas a un sospechoso, secuestrar un arma ilegal en un procedimiento en el que se buscaban drogas (Hasta dónde llegan los nuevos poderes de la Policía Federal. Clarín. Buenos Aires: clarin.com, 20 jun. 2001).

Los ejemplos siguientes presentan la interpretación de negación (3) y de elección libre (4). Se observa que podrian ser parafraseados por nadie y por cualquiera. Pero, de todos modos, la forma pronominal es la más frecuente en ambos casos, o sea, en el español argentino se prefiere nadie y cualquiera (con rasgo [+humano]) a ninguna persona o cualquier persona, aunque, comparando los dos contextos, se ve una presencia más acentuada del nombre en la construcción de elección libre.

(3) Ninguna persona fue enjuiciada por tales hechos (GASSINO, F. E.; BONIFACINO, E. B. Los 70. Violencia en la Argentina. Buenos Aires: Círculo Militar, 2001).

(4) Con el paso del tiempo, ese mito ha ido desapareciendo gracias a la llegada de un sinfin de servicios que han convertido al ciberespacio en un lugar

5 Como la cantidad de ocurrencias de una persona es muy alta, se analizó manualmente una muestra del $10 \%$ de las ocurrencias y se decuplicó el resultado.

6 En los ejemplos del CORPES XXI, se mantuvieron los mismos datos bibliográficos del corpus. 
emocionante para cualquier persona (IREGUI, L. A. ¿Conectados es mejor? Lanacion.com. Buenos Aires: lanacion.com.ar, 17 jul 2011.)

El CORPES XXI/Argentina no presenta casos de una persona en la interpretación genérica, lo que se corrobora con los resultados de los cuestionarios, que muestran un rechazo muy grande en un enunciado como Una persona se extinguirá un día $(4,3)$. Por otro lado, en contextos de referente inespecífico, de negación directa y de elección libre, la aceptabilidad de este nombre es casi tan buena como de la de los indefinidos alguien, nadie y cualquiera. Compárense los resultados de (5a) y (5b), (6a) y (6b) y (7a), (7b) y (7c).

(5) a. Cuando nos sentimos solos, debemos hablar con una persona. $(1,7)^{7}$

b. Cuando nos sentimos solos, debemos hablar con alguien. $(1,2)$

(6) a. No vi a ninguna persona en el despacho esta mañana. $(1,3)$.

b. No vi a nadie en el despacho esta mañana. $(1,2)$

(7) a. Estas herramientas le permitirán espiar fácilmente a cualquier persona. $(1,1)$

c. Estas herramientas le permitirán espiar fácilmente a cualquiera $(1,6)$

Los resultados revelan que persona, además de ser el más frecuente, es el nombre más adecuado para sustituir un indefinido en una referencia a un ser humano. Todos los demás nombres generales presentan una aceptabilidad inferior a éste.

El nombre general ser humano es el segundo más frecuente en el corpus. Los casos también se concentran en los textos no ficcionales, principalmente en los que se incluyen en los temas Ciencias y Tecnología (8) y Ciencias sociales, creencias y pensamiento (9). Pero, a diferencia de persona, este nombre se encuentra frecuentemente con una interpretación genérica, como se ve en los siguientes ejemplos:

(8) es imposible que un ser humano pueda hacer el ciento por ciento de las cosas que tiene que hacer (PAENZA, A. Matemática... ¿Estás ahî? Buenos Aires: Siglo Veintiuno Editores, 2005).

(9) En la Kabaláh existe la idea de que un ser humano es influido por su propia proyección astral, y viceversa (SZALAY, I. ¿Qué es la reencarnación? Buenos Aires: Kier, 2001).

El resultado anterior se alinea con un análisis diacrónico de la frecuencia de este nombre. Una consulta al Corpus del Español (DAVIES, 2002) revela que el nombre ser humano pasa a exhibir una frecuencia relevante a partir del siglo XX, especialmente en textos del registro académico, lo que se explica por la reducción

7 La aceptabilidad mejora si el nombre se encuentra modificado: Cuando nos sentimos solos, debemos hablar con una persona que nos escucha $(1,3)$. 
del empleo de hombre como genérico. Además, en los resultados del test de aceptabilidad, este nombre es muy aceptable en los enunciados genéricos de contextos científicos evaluados por los hablantes nativos, como se ve a continuación.

(10) Los seres humanos son seres vivos $(1,3)$

(11) El ser humano se extinguirá un día $(1,3)$

(12) El ser humano es consciente de su existencia. $(1,2)$

En los casos de negación, como (13) y de elección libre, como (14), se observa también un buen número de ocurrencias de ser humano, aunque menor que persona. A diferencia de la mayoría de los otros nombres generales, este resultado comprueba una buena integración de ser humano en diferentes construcciones empleadas en textos formales.

(13) Tanto desde el punto de vista psicológico como desde el energético, es evidente que ningún ser humano pertenece totalmente a un signo (CARUTTI, E. Ascendentes en Astrología. Segunda parte. Ciudad de Buenos Aires: Casa XI, 2001).

(14) Vio más allá que cualquier ser humano, estuvo miope durante un tiempo y le bajaron el pulgar varias veces para luego rescatarlo (Nebulosa Carina. Página/12. Futuro. Rosario: pagina12.com.ar/ diario/suplemento/futuro, 5 mayo 2007.)

Los casos de individuo en empleos indefinidos también se concentran en las obras no ficcionales, pero se distribuyen especialmente entre los textos del ámbito de Ciencias sociales, creencias y pensamiento y de Salud. Como los nombres anteriormente analizados, individuo surge en el contexto científico. Pero su frecuencia empieza a adquirir importancia en el siglo XVIII (CLAVERO, 2010). En el siglo XIX, comienza a aparecer en los diccionarios de la RAE como un nombre general para humano, lo que se realiza con la marca "familiar": "persona cuyo nombre y condición se ignoran o no se quieren decir" (DLC, 1884).

En la muestra, si bien se pueden encontrar casos de interpretación genérica, la mayor parte de las ocurrencias se da en empleos inespecíficos, lo que acerca este nombre más a los empleos de persona, que de ser humano y humano. A continuación, se pueden ver los ejemplos de cada interpretación, ambos del tema Salud:

(15) Una buena resiliencia comienza en la infancia. Un individuo tiene más facilidad de convertirse en resiliente si ha recibido buenos tratos (AMBROJO, J. C. Resiliencia. Los traumas no se curan a solas. Lanación.com. Salud. Buenos Aires: lanacion.com.ar, 25.04.2009).

(16) "El estudio permite aislar qué sistema falla y cuantificar cuán mareado está un individuo", agregó Videla (RÍOS, S. A. Mareos: frecuentes y mal diagnosticados. Lanación.com. Buenos Aires: lanacion.com.ar, 11.04.2004). 
En español, el nombre humano, como nombre general, se encuentra más frecuentemente en la forma plural (los humanos) ${ }^{8}$. De hecho, los enunciados Los humanos son seres vivos y Los humanos se extinguirán un dia, reciben una buena evaluación por los hablantes cordobeses, $(1,7)$ y $(1,9)$, respectivamente. En lo que se refiere a los empleos indefinidos, en el corpus estudiado, humano se encuentra principalmente en textos no ficcionales, sobre todo del tema Ciencias y Tecnología, como (17):

(17) Como estas tapas son de metal (hierro) muy pesado y son muy gruesas, si cupiera la posibilidad de que "cayeran" en el mismo pozo que están tapando, podrian obviamente lastimar gravemente a un humano (PAENZA, A. Matemática... ¿Estás ahî? Buenos Aires: Siglo Veintiuno Editores, 2005).

Como se ve en la Tabla 1, tanto las ocurrencias de individuo como de humano en contextos de negación y de elección libre son raras. En el caso de este último, el único ejemplo de negación es el que se lee en $(18)^{9}$ :

(18) Un árbol cae en medio del bosque, sin ningún testigo, sin ningún humano que presencie la escena (TABAROVSKY, D. Una belleza vulgar. Madrid: Caballo de Troya, 2001).

Al observar los casos de sujeto, se comprueba que, en cuanto a la distribución temática, este nombre presenta un comportamiento semejante a individuo, o sea, se distribuye especialmente entre los textos del ámbito de Ciencias sociales, creencias y pensamiento y de Salud, aunque con menor frecuencia. Las ocurrencias predominantes de sujeto son aquellas con interpretación específica, en que el nombre está modificado, sea por un sintagma adjetival, preposicional o por una oración de relativo. Por ello, si se tienen en cuenta las interpretaciones seleccionadas para este trabajo, no se observa este nombre en sintagma nominal genérico, solamente en interpretación inespecífica, como se ve en (19) y (20).

(19) Pero en una evaluación psicológica nunca podemos introducir todos los estímulos que se le pueden presentar a un sujeto en la vida cotidiana (PAZ, A. El valor predictivo de los psicotécnicos. Lanacion.com. Buenos Aires: lanacion.com.ar, 23.10.2011).

(20) ¿Por qué?, porque, por ejemplo, las malas condiciones socioeconómicas pueden afectar a un sujeto y, en esas circunstancias, una frustración o una pérdida afectiva, irritarlo hasta la violencia (JONAS, C. Encarar la violencia según sus causas. Página/12. Rosario: pagina12.com.ar, 31.05.2008).

8 En los datos del francés analizados por Mihatsch (2015, p. 65), la autora observa una preferencia de être humain en el singular y de humain en el plural.

9 Considerando el contexto con cualquier humano, se observa que en el ejemplo siguiente la oposición humano $\mathrm{X}$ animal es fundamental para la interpretación. La sustitución del sintagma destacado por cualquiera llevaría a la pérdida del rasgo [+humano]. Por este motivo, casos como este no se incluyeron en la cuantificación:

(i) -No te preocupes -dijo la pulga-, yo lo recordaré. Mi memoria es superior a la de cualquier humano (COVADLO, L. Criaturas de la noche. Barcelona: Acantilado, 2004). 
Conforme lo expuesto anteriormente, los elementos analizados suelen ser incluidos en la categoría de los nombres generales, ya que poseen contenido semántico mínimo, reciben definiciones semejantes en los diccionarios y se originan generalmente de contextos eruditos. Además, pertenecen a una categoría de unidades lingüísticas que en muchas lenguas dan origen a un proceso de pronominalización, como es el caso de pessoa en portugués o de insan en turco (HEINE; KUTEVA, 2004).

\section{2o. grupo: construcciones con otros nombres}

Considerando el número de ocurrencias del segundo conjunto de nombres que se pueden emplear en los diferentes tipos de SSNN indefinidos, el CORPES XXI/Argentina presenta el resultado de la Tabla 2:

Tabla 2 - Distribución de los otros nombres según el determinante que los antecede en SSNN indefinidos

\begin{tabular}{|l|c|c|c|c|c|}
\hline & un(a) & algún(a) & ningún(a) & cualquier & Total \\
\hline Alma & 17 & 0 & 0 & 1 & 18 \\
\hline Ciudadano & 8 & 0 & 2 & 5 & 15 \\
\hline Tipo & 8 & 2 & 0 & 0 & 10 \\
\hline Mortal & 0 & 0 & 0 & 5 & 5 \\
\hline Semejante & 3 & 0 & 1 & 0 & 4 \\
\hline Fulano & 1 & 0 & 0 & 0 & 1 \\
\hline $\begin{array}{l}\text { bípede / prójimo / quídam / } \\
\text { viviente }\end{array}$ & 0 & 0 & 0 & 0 & 0 \\
\hline Total & $\mathbf{3 7}$ & $\mathbf{2}$ & $\mathbf{3}$ & $\mathbf{1 1}$ & $\mathbf{5 3}$ \\
\hline
\end{tabular}

El nombre más frecuente de este conjunto es alma, lo que se puede relacionar con lo expuesto por la Nueva Gramática. Al analizar los términos de polaridad negativa, la NGLE denomina minimizadores a sustantivos de grupos nominales acompañados frecuentemente por el artículo indefinido un, una (ya que se usan como prototipos de valores minimos: haber un alma, valer un pepino, costar un centavo (NGLE, 2009, 48.7d, p. 3678)) y sostiene que la presencia de alma en este grupo podría vincularse con el origen nominal de nadie: "personas nacidas" (NGLE, 2009, 48.1c, p. 3632). De hecho, el corpus registra con mayor frecuencia la construcción no $V$ (ni) un alma, que ocurre mayormente en textos de ficción y entre estos en novelas. Véanse los ejemplos a continuación, en los que la construcción destacada se puede parafrasear por nadie:

(21) No habia un alma en Ravignani y Paraguay, a pesar de que no era tan tarde (FERNÁNDEZ DÍAZ, J. La segunda vida de las flores. Buenos Aires: Editorial Sudamericana, 2009). 
(22) No encontrará ni a un alma por allí, nadie arrea rebaños en medio de la guerra (DUJOVNE ORTIZ, A. Anita cubierta de arena. Buenos Aires: Alfaguara, 2003).

La única ocurrencia antecedida por cualquier también ocurre en un texto de ficción, en este caso en una obra de teatro (23). Como se observa, en todos los casos, no se emplea alma con el sentido relacionado a alguna sustancia espiritual o sobrenatural, sino con una equivalencia a persona.

(23) CREONTE: Desconfien. Esta ciudad es hija de contrabandistas. Desconfien. En Buenos Aires cualquier alma puede comprarse con dinero (HUERTAS, J. Antígonas: linaje de hembras. www.celcit.org.ar: celcit.org.ar, 28.02.2013).

De modo general, el nombre ciudadano ocurre más frecuentemente en el CORPES XXI acompañado por un adjetivo gentilicio (boliviano, brasileño, portugués, chino, etc.), presentando el concepto de "individuo de un Estado que posee derechos", tal como se ve en (24) y como el propio contenido de (25) ejemplifica. Este tipo de uso se concentra en los textos clasificados por el corpus en el tema Politica, economía y justicia.

(24) Un ciudadano estadounidense que viajó a la ciudad de Rosario para participar de un congreso de cardiología denunció que le robaron en el hall del hotel donde se alojaba 13 mil dólares que llevaba en un maletín (Rosario: engañan a un turista estadounidense y le roban un bolso con 13 mil dólares. Clarín.com. Buenos Aires: clarin.com, 03.05.2006).

(25) Un ciudadano es, como hemos señalado a lo largo de este trabajo, un sujeto que sintetiza la doble condición de individuo propietario y sujeto dueño de su voluntad en cuanto individuo libre (CIRIZA, A. ¿En qué sentido se dice ciudadanía de mujeres? Sobre las paradojas de la abstracción del cuerpo real y el derecho a decidir. In: HOYOS VÁSQUEZ, G. Filosofía y teorias politicas entre la critica y la utopía. Buenos Aires: CLACSO, 2007) ${ }^{10}$.

Sin embargo, considerando los criterios explicados en la metodología, la muestra argentina revela algunos casos en que este nombre se encuentra en SSNN indefinidos, con una pérdida del contenido semántico relativo a persona de derechos, o sea, casos en que ciudadano equivale más bien a "persona" o "individuo humano" y no le interesa al locutor si el referente es ciudadano de una u otra nación. Todas las ocurrencias se encuentran en textos no ficcionales y se distribuyen principalmente entre los temas Ciencias sociales, creencias $y$ pensamiento y Política, economía y justicia. Aunque el número de casos no es

10 En el ejemplo siguiente, de interpretación específica, el propio autor demuestra una equivalencia entre ciudadano y sujeto en la anáfora.

(i) Esta es la historia: un ciudadano escribe al Concejo de esta localidad costera preguntando por los planes municipales en el caso de una invasión de zombis. A los pocos días, el sujeto recibe una carta firmada por el director de Comunicación (DONADíO, M. Secretaría de Asuntos Imaginarios. Lanacion.com. Buenos Aires: Ianacion.com.ar, 17.07.2011). 
grande, se encuentran ejemplos en los diferentes tipos de interpretación, con interpretación genérica, inespecífica, en casos de negación y de elección libre:

(26) Antonio Juan Baseotto podrá seguir ejerciendo su derecho a decir misa. También podrá afirmar otra vez que un ciudadano puede ser ahogado en el mar por sus ideas (GRANOVSKY, M. Adiós al obispo que pidió el submarino. Página/ 12. Rosario: pagina12.com.ar, 19.03.2005.)

(27) el cardenal colombiano Alfonso López Trujillo, quien afirmó que "sólo un estado totalitario no respeta que un ciudadano se acoja a la objeción de conciencia" (Sigue la polémica por las bodas gay en España: el titular de la Junta de Galicia se sumó a los que se niegan a los casamientos. Clarín.com. Buenos Aires: clarin.com, 03.05.2005.)

(28) Creo que ningún ciudadano puede negarse a eso. Es un gran honor que el Presidente llame a una persona y ofrezca ese cargo", señaló Lorenzetti. (La renovación del máximo tribunal. Lorenzetti aseguró que sería "un gran honor" reemplazar a Vázquez en la Corte Suprema. Clarín.com. Buenos Aires: clarin.com, 02.09.2004).

(29) El descubrimiento, en la localidad bonaerense de Garín, de una instalación con suficiente capacidad tecnológica para escuchar a las comunicaciones de cualquier ciudadano, perteneciente a una empresa de telefonía que atribuye a la Secretaría de Inteligencia la propiedad del equipamiento con el cual se habrian interceptado ilegalmente lineas telefónicas de periodistas y de al menos una empresa editorial, debe obligar a las autoridades nacionales a intervenir, garantizando elementales derechos constitucionales que hoy parecen vulnerados. (Inteligencia sin control. Lanación.com. Buenos Aires: lanacion.com.ar, 02.12.2007.)

El contexto semántico de (29) es semejante al de enunciados testados con los hablantes de Córdoba. Los resultados de (30) muestran la buena aceptabilidad de persona y una mediana aceptabilidad de otros nombres generales como individuo y sujeto. Ser humano y humano, nombres más aceptables en contextos científico-académicos, no reciben buena evaluación. Por otro lado, ciudadano es bien aceptable por los hablantes, como se puede ver a continuación:

(30) Estas herramientas le permitirán espiar fácilmente a cualquier...
a. persona. $(1,1)$
b. individuo. $(2,2)$
c. sujeto. $(2,2)$
d. ser humano. $(2,7)$
e. humano. $(3,6)$
f. ciudadano. $(1,6)$ 
En cuanto al nombre tipo, en el español argentino se emplea preferentemente para referentes específicos y con modificadores (un tipo normal, un tipo que disfruta, etc.). Los casos que no llevan modificadores no determinan un predominio específico entre textos ficcionales o no ficcionales, pero entre los últimos están todos en novelas, lo que podría sugerir una tendencia de este nombre en este género ficcional - quizás un acercamiento a la modalidad oral. Se encuentran sobre todo ejemplos inespecíficos como (31) y (32) y se observa que, en muchas ocurrencias, tipo está empleado en contexto negativo, especialmente de violencia, como se ve también en los mismos ejemplos:

(31) Se puede apalear a un tipo por lo que sea, se lo puede reventar a balazos si se lo merece, pero no podés cortarlo en pedacitos día tras día, como querían hacer con Mauricio (ARGEMÍ, R. Siempre la misma música. Sevilla: Algaida, 2006).

(32) -Sí, claro, Colorado, podés hacerlo vos o conseguir a algún tipo que te simule un asalto, hace como que lo va a afanar y le pega dos tiros (CAPARRÓS, M. A quien corresponda. Barcelona: Anagrama, 2008).

Con relación al nombre mortal como equivalente a ser humano, se verifica que en los datos argentinos es utilizado principalmente en la forma plural y frecuentemente en la construcción el resto de los mortales. En los usos inespecíficos que conciernen a este trabajo, se encuentran pocos casos en la muestra, sin ningún predominio en textos de uno u otro tema. Todas las ocurrencias presentan una interpretación de elección libre, parafraseable por cualquiera o cualquiera persona. En los ejemplos, se ve que no hay una oposición explícita entre mortal $\mathrm{X}$ inmortal o divino.

(33) ¿Qué futbolista no soñó alguna vez con jugar en un gran estadio repleto de hinchas? Seguramente es uno de los tantos anhelos en la carrera de cualquier mortal (CAO, A. La curiosidad de jugar sin público. Clarín. com. Buenos Aires: clarin.com, 16.11.2006).

(34) De ahora en más y como a cualquier mortal, la felicidad en tu vida será de a iiratos!!! (WAINRAICH, S. Ser feliz me da vergüenza. Ser feliz me da vergüenza y otros cuentos. Buenos Aires: Sudamericana, 2008).

Las ocurrencias con semejante son muy raras en la muestra y todas se encuentran en textos ficcionales. Obsérvese la interpretación inespecífica de (35) y que en (36) el propio autor presenta la equivalencia entre nadie y ningún semejante.

(35) Ya no le temo, ahora conozco, lo estoy haciendo, qué es eso que se siente cuando se está frente a frente con un semejante... (MUJICA, H. Un espejo abierto a la vida. Bajo toda la lluvia del mundo. Buenos Aires: Seix Barral, 2008). 
(36) Sospecho, sin embargo, que nadie, ningún semejante podrá leerlo desde esta compartida superficie. (RAMPONELLI, A. La segunda temporada. Una costumbre de Oceanía. Buenos Aires: Ediciones Simurg, 2006).

Por último, se incluye en este conjunto la unidad fulano. Este nombre y las formas del paradigma (mengano, zutano, perengano y perencejo) se utilizan generalmente para reemplazar antropónimos. Pero sirven también para la referencia a un individuo cualquiera o, como destaca Casado Velarde (1999, p. 188-189), a personas "en general [...] cuando no se conoce, no se recuerda o no interesa mencionarlas por su nombre propio". Por ello, no se sorprende que gramáticos como Alvar y Pottier (1983), Coste y Redondo (1965) hayan incluido esas formas en el conjunto de los llamados pronombres indefinidos (cf. SATORRE GRAU, 2012).

En el siglo XVII, Correas (1984 [1627]) clasificaba como pronombres de formas como fulano, zitano y rroviñano y respectivas declinaciones, que serían usadas solamente para personas cuando "callamos los nonbres de las personas o porque se nos olvidaron, o no importa dezillos, o porque conviene callarlos" (p. 140). Fulano, sería, para el gramático, la forma más repetida y zutano una variante no apropiada de zitano (p. 140).

En el CORPES XXI/Argentina, la mayoría de las ocurrencias de fulano se encuentra sin determinante (37). Con la interpretación que concierne a este trabajo, se encuentra únicamente (38), extraído de una novela.

(37) En el ambiente artístico se hablaba de listas negras. Decian: "a fulano le pusieron la tapa” (KOSTZER, K. Pedestales de arena. www.celcit.org. ar: celcit.org.ar, 22.04.2013).

(38) ¿Qué se debe sentir cuando se le dice a uno que fulano de tal llamó veinte veces? ¿Desprecio? ¿Cierto placer narcisista al comprobar que nuestra parcelita de poder rinde lo suficiente como para que un fulano llame veinte veces? (FERREYRA, G. Piquito de oro. Buenos Aires: Seix Barral, 2011).

De acuerdo con lo expuesto anteriormente, al conjunto de unidades identificadas en este segundo grupo se podrían agregar nombres como bipede, prójimo, quídam y viviente, citados por gramáticos y lexicógrafos, pero ninguna de estas unidades ha sido identificada en los datos argentinos del CORPES XXI ${ }^{11}$.

11 Estas unidades pueden encontrarse en textos de otra variedad, con modificadores o con interpretación específica:

(i) ...abrazando la causa que abanderó un bípedo cantante llamado John Lennon: "Imagínate que no hay países, ni tampoco religiones" (JÁUREGUI, P. El centinela de las aves. El Mundo.es. Natura. Madrid: elmundo.es/natura, 10.05.2008) (España, interpretación específica, nombre con modificadores).

(ii) De modo que lo único que puede matar a quien beba de esa agua será la mala voluntad de un prójimo que le quite la vida de modo criminal (BURGOS, J. J. Don Juan de los Poderes. Venezuela: Universidad de Carabobo, 2003) (Venezuela, interpretación inespecífica).

(ii) Pero, en lo referente a este maldito excremento de roca que nos sostiene, ni remo, ni deshabitado, sino que ya era la finca particular de un quídam, reconocida y registrada en los dominios de Pintón y la muerte (NIEVA, F. El Cíclope. Madrid: Primer acto, 2009) (España, interpretación específica).

(iii) Había sido un viviente flaco, pero fue un globo en la muerte (GALEANO, E. La inflación. En Bocas del tiempo. Madrid: Siglo XXI de España Editores, 2004) (Uruguay, interpretación específica) 


\section{3er. grupo: construcciones no nominales}

Aparte de los nombres tratados anteriormente, la muestra del corpus permite identificar construcciones no nominales que se emplean con la misma función de los sintagmas indefinidos anteriores. Este es el caso de los ejemplos siguientes, en que tal o cual y no sé quién pueden parafrasearse por alguien o una persona. Ambos usos son, no obstante, raros en la muestra.

(39) ¿Tiene algo que ver el sexo, el casarse o no casarse o hacer el amor con tal o cual con estas nobilísimas misiones que acabamos de enumerar? (Elogio de la tolerancia. Lanación.com. Buenos Aires: lanacion.com.ar, 13.11.2005).

(40) Como si los astros, o no sé quién, o qué, me tuvieran preparada una emboscada (MARTÍNEZ, O. Días contados. Buenos Aires: Teatro Vivo, 2007).

En el caso de no sé quién, el uso se acerca al de no sé qué como proforma estudiada por Sánchez Jiménez (2014, p. 106), cuyos usos indefinidos ya se documentan en la segunda mitad del siglo XV. La forma para no humanos se encuentra fácilmente en los textos argentinos (ej.: Pero me daba no sé qué) y muestra los rasgos de lexicalización destacados por el autor. Las ocurrencias de no sé quién se emplean principalmente con una lectura específica para referentes no conocidos, como en (41):

(41) "No, no vayan a pensar que son pobre gente que está muerta de hambre. Ésos son infiltrados que les paga no sé quién" (PINTI, E. Que no se vaya nadie sin devolver la guita. Buenos Aires: Editorial Sudamericana, 2003).

Otras construcciones citadas por gramáticos y por lingüistas como más gramaticalizadas (un cómo se llama/llamó, un aquel, un don nadie, no importa quién) no se han identificado en la muestra. El número pequeño de los últimos ejemplos no permite relacionar estos casos con los discutidos por Haspelmath (1997) sobre la formación de pronombres. Quizás un análisis de corpus específicos de lengua oral de la misma variedad pueda revelar nuevos datos.

\section{Discusión De los Resultados}

Aparte de los pronombres indefinidos discutidos en cualquier gramática de lengua española, el análisis de la sección anterior permite identificar diferentes construcciones indefinidas para seres humanos. El cuadro siguiente presenta todos los elementos registrados en el CORPES XXI/Argentina. 
Cuadro 2 - Formas con rasgo [+humano] empleadas con el sentido de "una persona cualquiera" o "ninguna persona" en los datos del CORPES XXI/ Argentina

\begin{tabular}{|l|l|l|l|}
\hline $\begin{array}{l}\text { pronombres } \\
\text { indefinidos } \\
\text { para humanos }\end{array}$ & $\begin{array}{l}\text { nombres } \\
\text { generales }\end{array}$ & $\begin{array}{l}\text { nombres } \\
\text { comunes }\end{array}$ & $\begin{array}{l}\text { construcciones no } \\
\text { nominales }\end{array}$ \\
\hline $\begin{array}{l}\text { alguien } \\
\text { nadie } \\
\text { cualquiera }\end{array}$ & $\begin{array}{l}\text { humano } \\
\text { individuo } \\
\text { persona } \\
\text { ser humano } \\
\text { sujeto }\end{array}$ & $\begin{array}{l}\text { alma } \\
\text { ciudadano } \\
\text { mortal } \\
\text { semejante } \\
\text { tipo }\end{array}$ & $\begin{array}{l}\text { tal o cual } \\
\text { no sé quién }\end{array}$ \\
\hline
\end{tabular}

Considerando la distribución de los casos en los textos ficcionales y no ficcionales, se observa que, en general, los nombres generales persona, individuo, humano y ser humano y también ciudadano se encuentran preferentemente en textos no ficcionales, mientras formas alternativas como alma y semejante son más propias de textos ficcionales. La diferencia se puede explicar a partir de la importancia de los últimos nombres en contextos religiosos, mientras los primeros surgen y se difunden en contextos académico-científicos. Con relación al nombre persona, estudios anteriores han identificado que ocurre más frecuentemente en la modalidad oral, pero eso no se ha podido averiguar en el CORPES XXI, por la escasez de datos orales y por la inexistencia de datos de conversaciones espontáneas de la variedad diatópica elegida. De todas formas, el análisis muestra que persona, además de ser el más frecuente en el corpus, es el nombre más adecuado para sustituir un indefinido en una referencia a un ser humano.

En cuanto a los temas, se puede observar la tendencia de uso de algunos nombres y hacer algunas agrupaciones. En los textos pertenecientes a Ciencias sociales, creencias y pensamiento, es posible incluir, como unidades más frecuentes, las formas ser humano, individuo, sujeto y ciudadano. Si se trata de Ciencias y tecnología, se encuentran humano, ser humano y persona. En los textos de Política, economía y justicia, se observa mejor la presencia de persona y ciudadano. Por último, los textos del área de la Salud tienden a emplear individuo y sujeto. El corpus utilizado no presenta una clasificación fina en cuanto a la tipología textual, pero se cree que sería interesante observar en qué medida las formas estudiadas se distribuyen al considerar este criterio. De igual modo, valdria la pena averiguar, especialmente en el caso de los nombres generales, cómo se alternan en diferentes géneros jurídicos, ya que los autores del área suelen proponer una distinción teórica de tales nombres que no siempre se cumple en la práctica legislativa y forense (FERNÁNDEZ SESSAREGO, 2001).

Si se observan los casos de negación, se verifica que están concentrados casi todos en la función de sujeto, sea de negación directa (Ninguna persona presentó sintomas de intoxicación en los incidentes del lunes) o indirecta (Ni me dijo que ninguna persona hubiera llamado al diario para pedir más). Este resultado se explica por una inestabilidad de la posición de sujeto, que, conforme demostrado por otros estudios, favorece la entrada de nuevos elementos en la lengua y la referencia indefinida (AMARAL; RAMOS, 2014, p. 111). No obstante, destáquese 
que construcciones con cualquier también pueden presentar una interpretación de negación, como (42), en que cualquier equivale a ninguna, pero este tipo de uso es raro en la muestra. Asimismo, el uso del relativo quienquiera puede asemejarse a los casos de elección libre, como se ve en (43), pero como se realiza siempre con modificadores en el corpus, no ha sido tratado en este trabajo ${ }^{12}$.

(42) Es decir, la realidad no se deja poseer por cualquier persona; establece claves para que se la posea o se la viole (MARONNA, J.; PESCETTI, L. M. Copyright: plagios literarios y poder politico al desnudo. Barcelona: Plaza \& Janés, 2001).

(43) Sucedió hace tan sólo una semana, pero cualquier otra habría corrido enseguida a contárselo a quienquiera que se cruzase por su camino. (BRANCA, V. Hilos de seda. Con los pies desnudos. Buenos Aires: Ediciones Simurg, 2004).

\section{CONSIDERACIONES FinAles}

La NGLE clasifica y analiza los cuantificadores indefinidos, que también están presentes en los manuales de la lengua española. Pero, como se ha visto, desde hace siglos gramáticos, lingüistas y lexicógrafos señalan otras unidades que tendrian una función semejante a la de los indefinidos, o sea, la de permitir al hablante hablar de la existencia (o inexistencia) de un individuo cuyo nombre no sabe, no quiere o no puede nombrar, incluyendo la situación de cuando desea referirse a toda la clase de seres humanos. Este trabajo tuvo como objetivo analizar una muestra de datos de una variedad diatópica del español y ver cuáles son y cómo se distribuyen estas unidades en la lengua.

Los datos contemporáneos de Argentina permiten verificar que no solo los llamados nombres generales sino también otras unidades lingüísticas, más periféricas, presentan la función anterior. Entre estas, algunas están formadas por nombres comunes y otras por construcciones no nominales. El número de variantes estudiado no es exhaustivo, pero creemos que llega a ser representativo del conjunto de unidades que el español argentino dispone para hablar de la existencia o inexistencia de un ser humano, haciendo abstracción de su sexo y de otras características.

\section{ON THE INDEFINite constructions fOR human Beings in SPanish}

Abstract: The aim of this work is identify and analyze elements like persona, individuo, fulano, tal o cual, etc. in typical contexts of indefinite pronouns. The theoretical basis are the studies on this word class and on general nouns. The data used come from CORPES XXI and from acceptability test. The results show groups of elements formed by general nouns, other more peripheral nouns and

12 El indefinido quienquiera se utiliza casi siempre seguido de una oración relativa especificativa, pero hay casos en que, al igual que cualquiera y al contrario de lo que prescribe la NGLE, ocurre sin relativa. En el CORPES XXI este tipo de uso se registra más en el español mexicano. 
non-nominal constructions. The analysis also identifies trends based on the thematic classification of the corpus.

Keywords: Indefinite pronouns. General nouns. Spanish.

\section{REFERENCIAS}

ALVAR, M.; POTTIER, B. Morfología histórica del español. Madrid: Gredos, 1983. AMARAL, E. T. R.; RAMOS, J. M. Nomes gerais no português brasileiro. Belo Horizonte: Faculdade de Letras da UFMG, 2014.

AMARAL, E. T. R. Los nombres generales para humanos en español. Signo y Seña, n. 31, p. 1-22, 2017.

AMARAL, E. T. R.; MIHATSCH, W. Le nom français "personne" en comparaison avec le portugais brésilien "pessoa" et l'allemand "Person" - des noms en voie de pronominalisation? In: Actes du CMLF 2016 - 5e Congrès Mondial de Linguistique Française. Les Ulis: SHS Web of Conferences, v. 27, p. 1-17, 2016. Disponible en: <http://dx.doi.org/10.1051/shsconf/20162712015>. Acceso el: 22 dic. 2016.

CASADO VELARDE, M. Las formas fulano, mengano, zutano, perengano y su funcionamiento como ordenadores del discurso. In: GÓMEZ MANZANO, P.; CARBONERO, P.; CASADO VELARDE, M. (Coord.). Lengua y discurso - Estudios dedicados al Prof. Vidal Lamíquiz. Madrid: Arco Libros, 1999. p. 183-196.

CLAVERO, B. La máscara de Boecio: antropologías del sujeto entre persona e individuo, teología y derecho. Quaderni Fiorentini: Per la storia del pensiero giuridico moderno, v. 39, p. 7-40, 2010. Disponible en: <http://www.centropgm. unifi.it/quaderni/39/volume.pdf>. Acceso el: 22 dic. 2016.

CORREAS, G. Arte Kastellana. Introducción, edición y notas por Manuel Taboada Cid. Santiago de Compostela: Universidad de Santiago de Compostela, 1984[1627].

COSTE, J.; REDONDO, A. Syntaxe de l'espagnol moderne (enseignement supérieur). Paris: Société d'Édition d'Enseignement Supérieur, 1965.

DAVIES, M. Corpus del español: 100 million words, 1200s-1900s. 2002. Disponible en: <http://www.corpusdelespanol.org/>. Acceso el: 5 oct. 2016.

DE LA TORRE Y OCÓN, F. Nuevo methodo, breve, vtil y necesario para aprender a escribir, entender, y pronunciar las dos principales lenguas, española, y francesa. Madrid: Juan de Aritzia, 1728.

DLC - Diccionario de la lengua castellana. Real Academia Española. 12. ed. 1884. Disponible en: <http://web.frl.es/ntllet/SrvltGUILoginNtlletPub>. Acceso el: 7 abr. 2016.

DRAE - Diccionario de la Real Academia Española. Real Academia Española. Disponible en: <www.rae.es>. Acceso el: 22 dic. 2016.

FERNÁNDEZ SESSAREGO, C. ¿Qué es ser "persona” para el derecho? Derecho PUCP, v. 54, p. 289-333, 2001. 
FRANCIS, G. Labelling discourse: an aspect of nominal-group lexical cohesion. In: COULTHARD, M. (Ed.). Advances in written text analysis. London; New York: Routledge, 1994. p. 83-101.

GIACALONE RAMAT, A.; SANSÒ, A. The spread and decline of indefinite manconstrucctions in European languages: an areal perspective. In: RAMAT, P.; ROMA, E. (Ed.). Europe and the Mediterranean as Linguistic Areas: convergences from a historical and typological perspective. Amsterdam; Philadelphia: John Benjamins, 2007. p. 95-131.

GIACALONE RAMAT, A.; SANSÒ, A. (2011). L'emploi indéfini de homo en latin tardif: aux origines d'un "européanisme". In: FRUYT, M.; SPEVAK, O. (Ed.). La quantification en latin. Paris: Harmattan, 2011. p. 93-115.

HALLIDAY, M. A. K.; HASAN, R. Cohesion in English. 14. ed. London; New York: Longman, 1995[1976].

HASPELMATH, M. Indefinite pronouns. Oxford (Oxford studies in typology and linguistic theory): Clarendon, 1997.

HEINE, B.; KUTEVA, T. World lexicon of grammaticalization. Cambridge: Cambridge University Press, 2004.

HEINE, B.; SONG, K. On the genesis of personal pronouns: some conceptual sources. Language and cognition, v. 2, n. 1, p. 117-147, 2010.

HEINE, B.; SONG, K. On the grammaticalization of personal pronouns. Journal of Linguistics, v. 47, n. 3, p. 587-630, 2011.

KOCH, P.; OESTERREICHER, W. Lengua hablada en la Romania: español, francés, italiano. Madrid: Gredos, 2007.

LARRIVÉE, P. What nominal phrases are all about: the atypical case of indefinite pronouns with a determiner. Romanische Forschungen, v. 121, p. 3-19, 2009.

LEONETTI, M. El artículo. In: BOSQUE, I.; DEMONTE, V. (Dir.). Gramática descriptiva de la lengua española. Madrid: Espasa-Calpe, 1999. p. 787-890.

LEONETTI, M. Indefiniteness and specificity. In: HUALDE, J. I.; OLARREA, A.; O'ROURKE, E. (Ed.). Handbook of Spanish Linguistics. Oxford: Blackwell, 2012. p. 285-305.

MAHLBERG, M. English general nouns: a corpus theoretical approach. Amsterdam/Philadelphia: John Benjamins Publishing Company, 2005.

MARTÍNEZ, J. A. El pronombre II. Numerales, indefinidos y relativos. Madrid: Arco Libros, 1989.

MIHATSCH, W. La sémantique des noms généraux "être humain" français et allemands. In: MIHATSCH, W.; SCHNEDECKER, C. (Ed.). Les noms d'humains: une catégorie à part? Stuttgart: Steiner, 2015. p. 55-84.

MIHATSCH, W.; SCHNEDECKER, C. (Ed.). Les noms d'humains: une catégorie à part? Stuttgart: Steiner, 2015.

NGLE - Real Academia Española y Asociación de Academias de la Lengua Española. Nueva gramática de la lengua española. Madrid: Espasa, 2009.

NHUMA - Linguistique des noms d'humains, 2013. Disponible en: <http:// nomsdhumains.weebly.com/index.html>. Acceso el: 10 nov. 2016. 
REAL ACADEMIA ESPAÑOLA (2013). Corpus del español del siglo XXI (CORPES): descripción del sistema de codificación. Libros y prensa [Recurso de Internet]/Madrid: Real Academia Española, 2013. Disponible en: <http://www. rae.es/sites/default/files/CORPES_Sistema_de_codificacion_12_2015.pdf>. Acceso el: 3 may 2016.

SÁNCHEZ JIMÉNEZ, S. U. Entre lo uno y lo indefinido: aproximación diacrónica a las estructuras del tipo no sé qué en español. Valladolid: Ediciones Universidad de Valladolid, 2014.

SÁNCHEZ LÓPEZ, C. Los cuantificadores: clases de cuantificadores y estructuras cuantificativas. In: BOSQUE, I.; DEMONTE, V. (Dir.). Gramática descriptiva de la lengua española. Madrid: Espasa-Calpe, 1999. p. 1025-1128.

SATORRE GRAU, F. J. Pronombres con inventarios abiertos? Studia UBB Philologia, v. LVII, n. 1, p. 223-236, 2012.

SCHMID, H.-J. English abstract nouns as conceptual shells. Berlin; New York: Mouton de Gruyter, 2000.

Recebido em janeiro de 2017. Aprovado em setembro de 2017. 